\title{
LA DIMENSIÓN EXPRESIVO-EVALUATIVA EN ENTREVISTAS ORALES A ESTUDIANTES UNIVERSITARIOS ${ }^{1}$
}

\author{
MABEl GIAMmatTeO \\ (Universidad de Buenos Aires)
}

\begin{abstract}
RESUMEN
The aim of this work is to study some textual composition acts in relation with the so called expressive-evaluative area of language. The corpus of analysis is formed by interviews to universitary students about the beginnings of their career and their opinions about the different subjects. In the article, after a brief summary of the theoretical foundations of the investigation, we present the structural analysis of the interviews, divided into thematic parts, and seek to correlate the tcxtual composition acts involved in each part with the language functions -referential or emotive - they serve. Finally, we verify that the functions of the textual composition acts depend of the prevailing language function and, consecuently, we recognize that the same acts may be filled with different communicative values according with the language function they are associated.
\end{abstract}

\section{Introducción}

En este trabajo nos proponemos estudiar algunos actos de composición textual $^{2}$ tales como vacilaciones, frases inacabadas, reformulaciones, evaluaciones

Una primera versión de este trabajo fue leída en las Primeras Jornadas «De la teoría lingüística a la enseñanza del español», realizadas por el Instituto de Linguística de la Facultad de Filosofía y Letras de la Universidad de Buenos Aires, del 8 al 10 de junio de 1994. Este estudio forma parte de una investigación más amplia sobre la comprensión y producción lingüística de los estudiantes que ingresan en la Universidad, que dirigió la Dra. Ofelia Kovacci y llevaron a cabo los miembros de la cátedra de Gramática de la carrera de Letras de la Facultad de Filosofía y Letras de la Universidad de Buenos Aires. La investigación se realizó en el período 1992-1994 con un subsidio UBACYT FI-024. (Actualmente continúa como «Programa de lingüística aplicada a la comunicación académica en la Universidad» —UBACYT FI-078/ 1995-1997--).

2 E. Gülich y T. Kotschi $(1986,25)$ caracterizan los actos de constitución textual como «actos verbales que establecen formas particulares de composición textual ('Textgestaltung') como por 
y comentarios metadiscursivos ${ }^{3}$, en entrevistas orales realizadas a un grupo de estudiantes universitarios, luego del examen final de una materia. Nuestro interés se centra en determinar las funciones comunicativas que cumplen los distintos actos de constitución textual en una situación como la presentada y su relación con la denominada área expresivo-evaluativa del lenguaje.

Por to tanto, en primer lugar explicitamos brevemente los fundamentos teóricos y metodológicos del trabajo. Y luego, en el análisis de la estructura temática de las entrevistas, señalamos los actos de constitución presentes en cada una de las partes textuales que identificamos y correlacionamos las funciones comunicativas específicas que adoptan, con la función del lenguaje - referencial o emotiva- predominante. Por último, en las conclusiones, presentamos una interpretación, provisoria porque nos ceñimos al material analizado, de los resultados obtenidos en el análisis.

\section{Fundamentos teóricos}

\subsection{Los actos de composición textual}

El marco teórico en que se apoya nuestra consideración de los actos de composición textual lo constituyen los estudios de E. Gülich y T. Kotschi (1986) y el de Gülich (1986), que recogen la tradición más reciente de la lingüística textual, la teoría de los actos de habla y el análisis conversacional. En el enfoque de estos autores, que parten de una concepción interactiva del texto, «los fenómenos de constitución (textual) son el resultado de actividades verbales electuadas por los interlocutores susceptibles de ser interpretadas a partir de la estructura del texto» (Gülich y Kotschi, 1986, 18).

No es nuestra intención aquí hacer una presentación detallada de la teoría, sino sólo especilicar aquellos conceptos necesarios para el desarrollo del trabajo. Así, dentro de los actos de constitución textual analizados por Gülich y Kotschi (1986), constituyen una subclase especial, relevante para cl análisis que presentamos, las reformulaciones, cuya característica común es establecer una relación entre un «enunciado-fuente» y otro que lo reformula, a través de distintos marcadores de reformulación específicos para cada subtipo de acto. Actos de reformulación prototípicos son:

-la paráfrasis o relación de equivalencia entre dos segmentos de la estructura proposicional del texto;

- el refraseo o repetición de la estructura sintáctica y léxica de un enunciado;

ejemplo justificar, explicar, completar, repetir, parafrasear, resumir, acentuarm. (Ésta y todas las traducciones que aparecen en el trabajo son nuestras).

Seguimos la terminología utilizada en Gülich y Kotschi (1986) y Gülich (1986). Cf. el apartado 2.1., para una síntesis de los aspectos relevantes para nuestro trabajo, de la teoría de los actos de constitución textual planteada por estos autores. 
- la corrección o sustitución de un enunciado considerado «defectuoso» por otro (cf. Gülich y Kotschi, 1986, 30-31).

Por otra parte, también tendremos en cuenta, por la relevancia para la expresión del área emotiva que nos ocupa, otro subtipo de actos de composición textual estudiados por E. Gülich (1986), los denominados procedimientos de evaluasión y de comentario metadiscursivo ${ }^{4}$. Se trata de una categoría rica y variada, a menudo combinada con actos de reformulación, dentro de la cual Gülich reconoce tres subclases de enunciados:

- aquellos caracterizados por la ${ }_{i}$ resencia de expresiones calificativas,

- los que contienen expresiones metalingüísticas que remiten a la formulación del discurso,

- un conjunto de expresiones más o menos estereotipadas, genéricamente denominadas «atenuadores» (cf. Gülich, 1986, 236-235).

\subsection{La dimensión emotiva}

Para la consideración de la dimensión emotiva de los textos estudiados, partimos de los planteos de F. Danes, quien sostiene que todo discurso tiene un valor expresivo determinado, aunque no sea más que por defecto o ausencia (cf. 1987, 274). En consecuencia, los dos aspectos de la mente - cognitivo y cmotivo-, respectivamente manifestados por las funciones informativa o referencial y expresiva o emotiva, están siempre presentes en el lenguaje humano, aunque sus proporciones relativas varían según el tipo de texto y la situación.

El corpus que analizamos está formado por un conjunto de dieciséis entrevistas orales realizadas por docentes de la cátedra de Gramática ${ }^{5}$ de la Universidad de Buenos Aires, a alumnos que acaban de rendir la materia ${ }^{6}$. La situación se constituye en un entorno favorable para la aparición de «el estructurado complejo de emociones asociadas con una emisión (en la situación comunicativa dada)» (l.c.), porque dos de las cuestiones tratadas en la entrevista pertenecen a árcas temáticas relacionadas con la esfera subjetiva: las motivaciones para la elección de la carrera y sus opiniones acerca de la materia recientemente rendida. La primera pregunta evoca en la mayoría de los cntrevistados, o bien recuerdos de la infancia y la herencia cultural recibida, o bien la adolescencia e incertidumbre del último período de la escuela secundaria. La segunda, les plantea la necesidad de definirse mediante un juicio de valor

\footnotetext{
«Son por lo general los enunciados evaluativos/comentativos los que precisan cuál es el aspecto perturbador o problemático del enunciado-fuente» (Gülich, 1986, 236-235).

En el actual plan de la carrera de Letras, Gramática es normalmente la primera materia que cursin los alumos al ingresar a la facultad, luego de la aprobación del Ciclo Básico Común o curso preparatorio para la Universidad.

En todos los casos las entrevistas se pidieron a los alumnos como contribución voluntaria, luego de haber rendido el examen final y cuando tenían ya su nota colocada en la libreta; cs decir, que nada de lo que dijeran podía influir para la aprobación o no del examen.
} 
frente a un entrevistador no neutral ${ }^{7}$, que intenta distintos mecanismos de atenuación (cf. 3.3. infra) para tranquilizarlos.

Dado que en ambos casos los individuos son llevados a exponer el ámbito de sus experiencias y convicciones íntimas, nos interesa señalar que determinados mecanismos lingüísticos puestos en juego para proteger la esfera subjetiva son al mismo tiempo, como veremos más adelante (cf. 3.2. infra), manifestación de la función emotiva en el texto.

Por otra parte, es necesario aclarar que utilizamos el término 'emotivo' en un sentido muy amplio, dado que las manifestadas por los entrevistados no son de las del lipo denominado «emociones calientes» o más intensamente vividas por los individuos - tales como las que aparecen en el relato de un robo o accidente, por ejemplo-. Por el contrario, las que encontramos en los textos de nuestro corpus integran el campo de las llamadas «emociones frías» o «cognitivas» ${ }^{8}$, puesto que se trata de la verbalización de recuerdos lejanos, la manifestación de preferencias o la emisión de juicios de valor de índole personal.

Por último, si bien reconocemos que, como en todo lo referente al aspecto expresivo del lenguaje, los medios prosódicos o suprasegmentales son el soporte indispensable de la emotividad, el interés principal en este trabajo reside en el estudio de los recursos lingüísticos segmentales que manifiestan la dimensión expresivo-evaluativa en el discurso.

\section{La estructura temática de las entrevistas}

Aunque realizadas por distintos miembros de la cátedra de Gramática, todas las entrevistas tienen una estructura temática similar, ya que las preguntas de base habían sido previamente acordadas por los entrevistadores. En relación con las cuestiones tratadas, reconocimos en las entrevistas tres partes principales, coincidentes con los momentos inicial, intermedio y final, que, respectivamente, se ocupan de 1) las experiencias del comienzo de la carrera, 2) los motivos de la elección y 3) una evaluación de la materia que los alumnos acaban de rendir.

Por otra parte, como ya señalamos, sólo en las dos últimas, por la índole de las preguntas, está presente el aspecto emotivo. La primera parte, en cambio, es de neto predominio de la función referencial y todos los recursos linguísticos utilizados apuntan a que el entrevistador interprete correctamente la información transmitida por el entrevistado. Igualmente presentamos su análisis por dos razones: i) en principio, porque los actos de constitución textual que allí encontramos también están presentes en las otras dos partes en que predomina la lunción emotiva, aunque pasan a segundo plano, ya que en estos tramos de la

7 Dado que las entrevistas se realizaban luego de la aprobación de la materia, el entrevistador cra un miembro de la cátedra que no hubiera integrado la mesa de examen del alumno.

Para una presentación más detallada de la categorización y clasificación del campo emotivo según distintos autores, cf. Danes (1987, 275 y ss). 
entrevista lo puramente informativo queda como trasfondo de lo expresivo; y ii) en segundo lugar, por contraste, puesto que algunos de los actos de constitución textual utilizados con función informativa, al servicio de la función expresiva adoptan otros valores; así, por ejemplo, una repetición con sentido aclaratorio que aparece en la primera etapa de una de las entrevistas (cf. ej. (1) infra), al servicio de la función expresiva se convierte en un mecanismo dilatorio (cf. 3.3. infra). Es decir, que los mismos actos se llenan con distintos valores comunicativos, según con qué función del lenguaje estén asociados.

\subsection{La etapa inicial}

Las preguntas del comienzo se refieren al año de ingreso y a la cantidad de materias aprobadas hasta el momento de la entrevista ${ }^{9}$. Por lo general, estas preguntas iniciales no presentan dificultades para los alumnos y tampoco hacen aflorar cl aspecto emotivo del lenguaje. En ellas los entrevistados se esfuerzan por ser comprendidos y los actos de composición textual que utilizan están principalmente al servicio de la función informativa.

Desde este punto de vista, en esta parte de la entrevista se encuentran numerosas paráfrasis (cf. supra 2.1.) que pueden presentarse bajo cualquiera de sus tres formas: a) expansión, b) reducción, o c) variación (cf. Gülich y Kotschi, 1987, 39-42), así como también refraseos y correcciones. Dado que no hay un esquema único para cursar la carrera, todos estos actos de reformulación apuntan a poner en claro:

a) lo que los entrevistados sienten que el entrevistador no ha comprendido bien. Estas reformulaciones del enunciado propio, que tienen origen en el hablante mismo, son las que Gülich y Kotshi denominan de auto-reformulación auto-iniciada ${ }^{11}$ :

\footnotetext{
A: $\mathrm{Y}$ en el primer cuatrimestre rendi, eh .. hice, el Ciclo Básico. (Corrección)[5] $]^{11}$
}

9 En algunos textos esta parte puede reducirse a la sola pregunta acerca del año de ingreso; pero en otros, las explicaciones acerca de equivalencias o detalles conexos pedidos por el entrevistador para ampliar el tema, pueden llevar a los entrevistados a demorarse varios turnos en las respuestas.

It) «un locutor puede reformular uno de sus propios enunciados (auto-reformulación) o el enunciado de un interlocutor (hétero-reformulación)». Por otra parte «la actividad de reformulación puede estar iniciada tanto por el locutor que produce el enunciado reformulador (auto-iniciativa) como por aquel al que él se dirige (hétero-iniciativa)» (Gülich y T. Kotschi , 1986, 50).

it Para las convenciones de transcripción, cf. Apéndice.

Las palabras en bastardilla son las que fundamentan nuestra interpretación de los ejemplos. Los números remiten al número de orden correlativo con que fue designado en el corpus, el texto al que pertencen: v.gr. [ 1 ] = ej. tomado del texto 1 del corpus. 
b) lo que reformulan compelidos por una repregunta del entrevistador -hétero-reformulación hétero-iniciada-

(2) E: ¿Y de la carrera de Letras, cuántas tenés?

(...)

A: Bua..¿cuántas tengo aprobadas como Letras? (...) (Paráfrasis de expansión)[1]

Además, tratándose de una parte de la entrevista donde la cooperación entre los hablantes tiene como meta la trasmisión de información, abundan las señales lingüísticas o marcadores de ratificación ${ }^{12}$, que manifiestan la comprensión de una y otra parte:

Entrevistadores: ah.. [1] está [1] bueno [2] claro [12] ahá [5] [7] bueno..perfecto

[10] buah...perfecto [11]

Alumnos : claro..sí, exactamente [1] sí, sí, sí [1] [7] [13] uhú: [3] exacto [13] [15]

A veces, además del marcador, la ratificación retoma alguna(s) palabra(s) del turno anterior. En el ejemplo (3), el alumno rectifica al entrevistador con una de las palabras empleadas por éste, quien en el turno siguiente la vuelva a utilizar con sentido ratilicatorio:

(3) E: ¿No terminaste Derecho?

A: Si, terminé...sí.. (...) (Paráfrasis de reducción)

E: Ah.terminaste. (Refraseo) Ah, bueno, doctora. [1]

En (4), el refraseo con adaptación de la persona con que el alumno responde al entrevistador, cuenta también como ratificación:

(4) E: ¿Éste es tu primer final?

A: Éste es mi primer final. (Refraseo)[6]

Como dijimos, este empleo de los actos de constitución textual apuntando fundamentalmente a lograr la comprensión entre los hablantes, no desaparece completamente en el resto de la entrevista, sino que se hace presente siempre que es necesario ratificar o rectificar información. Pasa, no obstante, a segundo plano, desplazado por el uso expresivo de estos recursos que predomina en las otras partes.

12. Se trata de usos ligados a la función fática del lenguaje, cf. C. Fuentes Rodríguez (1993), especialmente para bueno, bien y pues bien utilizados en «respuestas confirmativas o de aceptación» (1.c., 208). 


\subsection{La etapa intermedia}

En la segunda etapa de la entrevista, que ya penetra en la esfera subjetiva del entrevistado, comprendemos dos pares de pregunta-respuesta muy relacionadas: los motivos de clección de la carrera y las expectativas futuras al respecto.

Frente a ambas preguntas aparecen comentarios metadiscursivos de tipo calificativo (cf. supra 2.1.) que manifiestan, mediante distintas variantes cxclamativas, reacciones «emotivas» de sorpresa y desconcierto por parte de los entrevistados. De acuerdo al esquema planteado por Gülich y Kotschi (1986) y aplicado al análisis de este subtipo de actos de constitución textual en particular por Gülich (1986), los ejemplos hallados son todos hétero-evaluaciones autoiniciadas, ya que con ellas los entrevistados apuntan a explicitar su propia opinión acerca de la pregunta formulada por el entrevistador:

(5) Me gusta..iQué sé yo...! iQué pregunta! [3]

(6) ¡Ay..qué pregunta difícil! [13]

(7) iQué preguntas dificiles...! ¿Qué expectativas...?[2]

También encontramos formas de contenido equivalente, pero sin énfasis:

(8) ...Es dilícil explicarlo...Es difícil (...) [4]

Estas preguntas, evidentemente no esperadas por los entrevistados, los sacan del estado de comodidad y tranquilidad en que se venía desenvolviendo la entrevista y los llevan a desarrollar mecanismos lingüísticos de respuesta que actúan como «escudo protector» del ámbito subjetivo que sienten invadido, a la vez que son manifestación de la función expresiva. Los más típicos son:

-multiplicación de prefacios o marcadores de inicio de respuesta, vacíos de significación:

(9) $\mathrm{E}: \mathrm{Y}$ ¿de dónde te vino la vocación para Letras, esa que tuviste desde sicmpre?

Büno..en realidad...quizá..este..(...) [1]

En este uso inicial, según Fuentes Rodríguez (1993) bien y, en nuestros ejemplos del dialecto rioplatense, bueno ${ }^{1.3}$ «surge tras un pensamiento desordenado de muchas cosas que se van a organizar, o recogiendo el turno de la palabra: yo empiezo a responder». Es un elemento fático, como organizador macroestructural, y para romper la violencia del inicio» (l.c., 213).

13 En el dialecto rioplatense actual prácticamente ha desaparecido de la lengua oral coloquial, cl conector pragmático bien, con la consecuencia de que bueno ha asumido gran parte de sus funciones discursivas. 
- utilización de prefacios combinados con otros elementos introductorios de la respuesta:

Bueno..hubo varias razones (...)[2]

- empleo de distintos elementos que enmarcan la respuesta:

- un elemento introductorio que a modo de refraseo encierre la respuesta:

(11) Bueno.por empezar, a mí me gusta la literatura, por empezar. [2]

- un topicalizador ${ }^{14}$, que puede estar tomado de la misma pregunta, que funciona como encuadre inicial para la respuesta:

(12) E: (...) ¿Qué expectativa tenés con respecto a la carrera?

A: Bueno.. con respecto a la carrera eh... (...) (Refraseo)[8]

- un especificador del valor de verdad de la aserción ${ }^{15}$-inicial (13) o siguiendo al prefacio (14) - mediante el cual el hablante busca destacar la condición de sinceridad de su respuesta:

(13) La verdad, que no sé. [6]

(14) Bueno.. la verdad, que no sé qué expectativas... [10]

- una auto-repregunta del entrevistado:

(15) E: ¿Qué expectativas tenés sobre la carrera de Letras?

A:i Expectativas?! (Paráfrasis de reducción) [7]

Un buen ejemplo, por la extensión del procedimiento es (16), en que el hablante, merced a estos mecanismos dilatorios, logra demorar seis turnos su respuesta. Ésta, luego de un tajante no sé inicial, se apoya principalmente en dos mecanismos: la construcción aproximativa introducida por como que y el refraseo diferido, que, a diferencia del inmediato que se establece entre enunciados contiguos, se da «entre dos enunciados separados uno del otro por otros enunciados intercalados» (Gülich y Kotschi, 1987, 57).

(16) E: Bueno.. y ahora que estás en la carrera, ¿qué esperás de la carrera?

14 «La topicalización consiste en la selección de un elemento de la oración como 'tópico' de la oración, lo que se marca o bien asignando al elemento topicalizado la posición inicial o si ésta le corresponde de todos modos, separándolo del resto de la oración por una pausa o anteponiéndole expresiones 'topicalizadoras' como en cuanto, hablando de, etc.» (Contreras, 1978, 98).

15 Se trata de formas con valor adverbial cuya función oracional es reforzar el valor de verdad del modus (tanto actitud del hablante) de la aserción, cf. Kovacci (1980-1981, §2). 
A: Ehhh...¿Quéee, qué espero? (Paráfrasis de reducción) ¿Qué espero a nivel futuro, qué voy a hacer yo con ella? (Paráfrasis de expansión)

E: Sí, tus expectativas. (Ratificación y paráfrasis de variación)

A: Mis expectativas...(Refraseo, con adaptación de persona)

E: De la carrera... (Completamiento)

A: No sé. Como que estoy medio en el aire en ese sentido, como que (...), expectativas...este..¿QQué...(...)? (Refraseo diferido) [3]

En casos menos extremos que el anterior, al mismo tiempo que defensores de la intimidad que tarda en aflorar, estos mecanismos dilatorios permiten a los alumnos mantener el turno mientras realizan una rápida introspección en busca de una contestación que resulte fundamentada y convincente para su interlocutor. Ésta generalmente involucra un turno largo, de naturaleza argumentativa, en el que ya muchos abandonan la sorpresa inicial y dan razones sobre su elección y expectativas futuras de la carrera. En otras respuestas, en cambio, de las que nos ocuparemos particularmente, la argumentación no avanza desde la JUSTIFICACIÓN hasta la CONCLUSIÓN ${ }^{16}$ por una vía de razonamientos sucesivos. Por el contrario, en estas respuestas, la argumentación se presenta circular, de modo que las categorías mencionadas -JUSTIFICACIÓN Y CONCLUSIÓN - se identifican y el razonamiento se impregna de elementos subjetivos que manifiestan la índole 'emotiva' del discurso. A modo de ejemplo analizaremos este tramo de la entrevista en dos de los textos del corpus:

(17) Ehhh...no sé muy bien, como que siempre pensé que iba a estudiar Letras.

Siempre me gustóooo eso. Me..me gustó leer. Me gustóooo la lingüística y...ya hace años que dije que iba a estudiar letras. Era como...así, como ya he dicho. Me gusta..¡Qué sé yo !..jQué pregunta! [3]

En el ejemplo anterior, el alumno comienza con una manifestación acerca de su imposibilidad de expresar razones fundadas de su elección - no sé muy bien-, que enseguida contrarresta con una afirmación enfática de su preferencia, remarcada por el rasgo prosódico del alargamiento vocálico — me gustóooo-. Este concepto se reitera con una paráfrasis de amplificación - me gustóooo eso. Me...me gustó leer. Me gustóooo la lingüística-. Por otra parte, la única LEGITIMIDAD — categoría de la argumentación que 'autoriza' a alguien a llegar a una conclusión determinada (cf. van Dijk, 1978, 159) - en que se apoya la causa, es que se presenta como una decisión tomada por el sujeto hace mucho tiempo - siempre pensé, siempre me gustóooo, ya hace años que dije que iba a estudiar - Hacia el final, el cambio del tiempo verbal al presente - Me gustaremarca la continuidad en la decisión. Finalmente una paráfrasis retoma el argumento inicial acerca de la imposibilidad de dar razones sobre los motivos de

16 Para las catcgorías básicas de la argumentación seguimos a van Dijk (1978, 158-163). 
clección de la carrera —iQué sé yo! - y la argumentación remata con una expresión de asombro - i Qué pregunta! - Se trata, pues, de una argumentación cíclica que, luego de un escarceo de fundamentación, se diluye en una manifestación 'emotiva'.

De tipo semejante a la anterior es la respuesta a la pregunta sobre las expectativas en el ejemplo (18):

(18) La elegí porque...porque me gusta. Es una respuesta muy simple pero es lo que más me gusta y es a lo que me quiero dedicar. Y porque quiero escribir y me pareció que me podía servir para perfeccionarme y para, para tener mucha más cultura en todo lo que fuera literatura....En realidad yo lo que quiero es escribir, no hacer docencia ni nada de eso. Y me pareció que me podía servir muchísimo. porque me gusta, desde que tengo, desde chiquita que leía y leía y pienso que, iqué sé yo!, es una forma de vida Letras. No sé, me encanta. [13]

En (18) la respuesta comienza con una afirmación con titubeos, que basa la elección en una decisión personal - La elegí porque.porque me gusta-, a la que el alumno justifica con una comentario metadiscursivo de tipo calificativo (autoevaluación auto-iniciada) —es una respuesta muy simple - y una paráfrasis de su alirmación inicial del tipo ampliación — pero es lo que más me gusta-Recién en su tercer intento de JUSTIFICACIÓN logra presentar una serie de razones bien lundadas de su elección — es a lo que me quiero dedicar, (...) quiero escribir (...), me pareció que me podía servir para perfeccionarme y para, para tener mucha más cultura (...), no hacer docencia (...), me pareció que me podia servir muchísimo-. Finalmente, con un refraseo enfático vuelve al argumento inicial $-y$ porque me gusta-, luego del cual la explicación se carga de 'emotividad' tal como distintos elementos lo señalan: la exclamación: ¿Qué sé yo!, parafraseada luego con no sé y scguida de una construcción marcada por el uso de un hipérbaton: es una forma de vida Letras y, por último, una paráfrasis del argumento inicial que, como en (17), vuelve cíclica la argumentación: $\underline{\text { Me encanta. }}$

Por último, inevitablemente, en todos los casos en que los entrevistados confiesan que su inclinación por la carrera o su expectativa futura tiene que ver con la vocación de escribir, al indagar el entrevistador en este sentido, tiñen sus respuestas de afectividad. Como elementos caracterizadores desde el punto de vista lingüístico encontramos en estas respuestas interjecciones, elementos léxicos que señalan incertidumbre, pronombres indefinidos, especificadores del valor de verdad (19) y refraseos que enmarcan la secuencia (20) (cf. ejemplo (11) supra).

(19) E: ¿Podrías darnos un panorama de tu poesía?

(...)

A: $;$ Ay...!

E: ¿Por qué escribís?

A : No sé porque escribo (...) No sé dar respuestas así. (...) 
E: ¿Por qué elegiste la división estrófica?

A: Ay ...la verdad, no sé.., [10]

(20) E: ¿Escribís?

(...)

A: De todo. Ahora poesía.(...). Escribía cuentos... De todo. [6]

\subsection{La etapa final}

El tramo final de la entrevista mantiene o vuelve a colocar a los entrevistados, según los casos, en el plano emotivo; pero ahora en el área evaluativa, puesto que se les pide una opinión acerca de la materia que han rendido.

Nuevamente aparecen los mecanismos de dilación de respuesta antes detectados. E igualmente tienen la doble función de proteger la esfera subjetiva de la opinión personal y de dar tiempo para elaborar una respuesta «adecuada» a una pregunta que, a pesar de los esfuerzos del entrevistador por suavizarla, sienten como «comprometida» ${ }^{17}$.

Por lo tanto, caracterizan particularmente esta parte de la entrevista, diversos mecanismos de atenuación (cf. supra 2.1.), tanto en la introducción de la pregunta por parte del entrevistador, que apunta a garantizar al alumno la más absoluta libertad de expresión, como en las respuestas de los entrevistados. De parte del entrevistador estos procedimientos descansan fundamentalmente en elementos léxicos y de construcción (21) y (22), aunque no se descartan otros, como el uso de diminutivos, típico recurso de atenuación (23):

(21) Bueno, y de esta materia, fuera de...tu nota, no depende en absoluto de la opinión... [3]

(22) (...) Te aclaro que lo que digas...no puede ser usado en tu contra ${ }^{18}$ [1. 10].

(23) Bueno, te hacemos otra preguntita... (...) [11]

Ante el pedido de evaluación de la materia, los entrevistados sienten nuevamente una intromisión en su esfera personal, ahora en lo tocante a sus opiniones. En consecuencia, aparecen reacciones 'emotivas' de desconcierto:

(24) ¿Cómo qué evaluación? (Repregunta y exclamación)

(...)

Son difíciles las preguntas [9] (hétero-evaluación auto-iniciada (Cf, supra 3.2.))

17 A tal punto que uno de los entrevistados liega a hacer explícito que acepta responder sólo porque ha aprobado el examen:

A: Terminé, aprobé..Sí.., sí, si no, no te daba la encuesta. [15]

18 En (22) el efecto de atenuación se apoya en el recurso retórico de la ironía, que surge de la utilización, con sentido contrario, de una frase corriente en el lenguaje jurídico. 
Igualmente encontramos los mismos mecanismos dilatorios de la respuesta registrados anteriormente:

(25) E: De la materia, ahora (?...) ¿qué evaluación hacés?

A: De la materia... Bueno. (...) (Refraseo enmarcador) [1]

(26) E: (...) ¿Qué evaluación podés hacer de esta materia?

A: De esta materia.., de gramática,...eh...(...) (Refraseo y paráfrasis de expansión) [2]

En el desarrollo de la respuesta, los entrevistados ponen en juego una serie de mecanismos de atenuación que buscan suavizar la crítica, que, en la mayor parte de los casos, no se introduce directamente, sino precedida de un elogio.

(27) (...) Se dió mucho. mucho contenido (...)...y creo que estuvo muy bien dada, (...) tal vez algunos criterios no es que me parecieron mal, pero...(...) es una opinión mía. [8]

En (27) el entrevistado comienza elogiosamente y luego presenta sus objeciones, matizadas por elementos que manifiestan una modalidad dubitativa -tal vez, no es que me parecieron- y cierra con un comentario que remarca, léxicamente, que lo dicho pertenece a su esfera personal de opinión.

Otras veces se van introduciendo objeciones o reparos de modo paulatino, mediante una gradación. En (28) el hablante introduce primero un elogio, luego con pero coordina una primera objeción y finalmente añade una sugerencia:

(28) (...) Estoy. Bueno...muy satisfecha (...); pero lo que considero es que son muchos contenidos (...) y, esteh... una sugerencia.. (...). [1]

Por último, también los alumnos recurren a diminutivos para la presentación de sus críticas:

(29) Me pareció que falta un poquito eso. [2]

\section{Conclusiones}

Según la función del lenguaje dominante y las intenciones comunicativas de los interlocutores, en cada uno de las tres partes identificadas en las entrevistas reconocimos funciones específicas para los actos de composición textual que las caracterizan.

En la etapa inicial predomina la función informativa y los actos de composición textual empleados — paráfrasis, correciones y reformulaciones de distintos tiposfuncionan como un mecanismo cooperativo, que apunta a resolver los problemas de comprensión que se presentan entre los interlocutores. En el resto de la entrevista 
estos mecanismos no desaparecen, pero quedan en segundo término, especialmente en aquellos tramos en que la dominante es la función expresiva.

En la etapa intermedia por lo general se introduce, en mayor o menor medida según los casos, la función expresiva. La manifestación de la 'emotividad' en las respuestas es puesta en juego por la sorpresa que genera una pregunta no esperada. Como consecuencia, los alumnos se valen de una serie de recursos lingüísticos de tipo dilatorio - tales como prefacios, introducciones, encuadres, topicalizadores, auto-repreguntas, especificadores del valor de verdad, etc.- , que constituyen actos de composición textual cuya función es proteger el mundo subjetivo, al mismo tiempo que conservar el turno mientras se elabora la respuesta, que se siente dificil porque obliga a verbalizar contenidos propios del área emotiva, que tienen más que ver con preferencias personales y juicios de valor, que con razonamientos.

Las explicaciones que dan los alumnos acerca de su decisión sobre la elección de la carrera y expectativas al respecto, cubren un amplio espectro que va de la pura manifestación 'emotiva' — vg. exclamaciones — hasta la argumentación; pero aún ésta, en buena parte de los casos, se carga de elementos que manifiestan distintos matices de lo expresivo. Si bien encontramos argumentaciones sólidas y bien desarrolladas ${ }^{19}$, muchas de las presentadas son de tipo circular, puesto que simulan desarrollarse a través de razonamientos más o menos fundados, pero en la conclusión vuelven al punto inicial, sin que ninguno de los argumentos presentados haya sido realmente empleado en su elaboración.

En la etapa final, en muchas de la entrevistas se mantiene el uso de la función expresiva, pero con sentido evaluativo. Aunque varios de los recursos y funciones ya identificados se conservan, surgen como característicos una serie de mecanismos de atenuación, cuya función específica varía según cuál de los interlocutores los utilice. Empleados por el entrevistador, estos recursos tratan de garantizar al entrevistado la máxima libertad de expresión posible; de parte de este último, en cambio, buscan suavizar las objeciones presentándolas bajo la modalidad de la opinión personal .

Por lo que conocemos, no existen muchos estudios que tengan en cuenta el factor «emotivo» en el español coloquial, desde la perspectiva en que lo abordamos en el presente trabajo. Por supuesto, las conclusiones arriba presentadas se limitan al corpus analizado y serán necesarios estudios sucesivos que den cuenta de múltiples factores no contemplados aquí. En este trabajo, de carácter inicial, hemos intentado hacer un aporte para desentrañar algunos de los mecanismos lingüísticos, que buscando encubrir, resultan clara manifestación del

19) Como el objetivo de este trabajo es estudiar la relación entre ciertos actos de composición textual y el área expresivo-evaluativa del lenguaje, aquellas argumentaciones en que sólo encontramos exposición de razonamientos y predominio de la función informativa no han sido tenidas en cuenta. 
«estructurado complejo de emociones» (Danes, 1987, 274), que surge en ciertas situaciones comunicativas en que los hablantes se ven compelidos a expresar sus preferencias o a hacer evaluaciones personales acerca de circunstancias determinadas del mundo que los rodea. A partir del análisis de la relación de algunos actos de composición textual con el área expresivo-evaluativa del lenguaje, así como de ciertas características de la argumentación relacionadas con la función emotiva, esperamos haber hecho alguna contribución al esclarecimiento de la relación entre expresividad y lenguaje, en una lengua determinada.

\section{APÉNDICE : CONVENCIONES DE TRANSCRIPCIÓN}

$\begin{array}{ll}\mathrm{E}=\text { cntrevistador } & \\ \mathrm{A}=\text { alumno } & \\ , & \text { pausa muy corta. } \\ . . & \text { pausa corta. } \\ \ldots & \text { pausa mediana. } \\ \text { aaaa } & \text { alargamiento de sonidos. } \\ \text { dilícil } & \text { acentuación notoria. } \\ (\ldots ?) & \text { fragmento incomprensible. } \\ (\ldots) & \text { fragmento dejado de lado. } \\ \text { E Bucno, así que decidiste eso. } & \text { emisiones simultáneas. }\end{array}$

\section{Referencias bibliográficas}

CONTRERAS, H. (1978), El orden de palabras en español, Madrid, Cátedra.

DANES, F. (1987), «Cognition and emotion in discourse interaction: A preliminary survey of the field». Proceedings of the IVth International Congress of Linguistics, Berlin, Akademieverlag, 272-291.

DIJK, T. VAN (1978), La ciencia del texto, Barcelona-Buenos Aires, Paidós.

FUENTES, C. (1993), «Comportamiento discursivo de bueno, bien, pues bien», Estudios de Linguiística 9, 205-221.

GÜLICH, E. (1986), «'Soûl' c'est pas un mot très français». Procédés d'évaluation et de commentaire métadiscursifs dans un corpus de conversations en 'situations de contact'». En Cahiers de Linguistique Française 7, Université de Genève, 231-258.

GÜLICH, E. ET KOTSCHI, T. (1987), «Les actes de reformulation dans la consultation La dame de Caluire». En P. Bange (ed.), L'analyse des interactions verbales. La dame de Caluire: une consultation, Berne /Francfort/New York/Paris: Lang., 15-81. (= Sciences pour la communication 18).

KOVACCI, O. (1980-1981), «Sobre los adverbios oracionales». En Homenaje a Ambrosio Rabanales, BFUCh XXXI. Reproducido en sus Estudios de Gramática Española, Buenos Aires, Hachette, 1986. 